\title{
Quantum Entanglement as a Consequence of a Cantorian Micro Spacetime Geometry
}

\author{
Mohamed S. El Naschie* \\ Department of Physics, University of Alexandria, Alexandria, Egypt \\ E-mail: ${ }^{*}$ Chaossf@aol.com \\ Received June 20, 2011; revised July 18, 2011; accepted August 2, 2011
}

\begin{abstract}
Building upon the pioneering work of J. Bell [1] and an incredible result due to L. Hardy [2] it was shown that the probability of quantum entanglement of two particles is a maximum of 9.0169945 percent [2]. This happens to be exactly the golden mean $\phi$ to the power of five $\left(\varphi^{5}\right)$ [3-7]. Although it has gone largely unnoticed for a long time, this result was essentially established independently in a much wider context by the present author almost two decades ago [3-6]. The present work gives two fundamentally different derivations of Hardy's beautiful result leading to precisely the same general conclusion, namely that by virtue of the zero measure of the underlying Cantorian-fractal spacetime geometry the notion of spatial separability in quantum physics is devoid of any meaning [7]. The first derivation is purely logical and uses a probability theory which combines the discrete with the continuum. The second derivation is purely geometrical and topological using the fundamental equations of a theory developed by the author and his collaborators frequently referred to as E-infinity or Cantorian spacetime theory [3-7].
\end{abstract}

Keywords: Hardy’s Quantum Entanglement, Golden mean, Cantor sets, Fractal Spacetime, E-Infinity Theory, Quantum Mechanics, J. S. Bell

\section{Introduction}

In his equally ingenious as it is beautiful Gedanken experiment, Hardy showed using orthodox quantum mechanics and Dirac's notation that a two particles entangled state will have a maximum probability of $(5 \sqrt{5}-11) / 2 \quad$ [2]. In particular for a basis state $| \pm\rangle_{\mathrm{i}}$ for two particles 1 and 2 the entanglement is given by the Schmidt decomposition [2].

$$
|\psi\rangle=\alpha|+\rangle_{1}|+\rangle_{2}-\beta|-\rangle_{1}|-\rangle_{2}
$$

Here $\alpha$ and $\beta$ are two real constants satisfying [2]

$$
\alpha^{2}+\beta^{2}=1
$$

Proceeding in this way Hardy's subtle analysis arrives at a general expression for nonlocality which need not be associated with spin but rather any other measurable quantity as in our two-slit experiment for example $[3,4]$. Using Hardy's notation the expression is [2]

$$
\gamma=\left(\frac{(|\alpha|-|\beta|)|\alpha \beta|}{1-|\alpha \beta|}\right)^{2}
$$

(*) Distinguished Fellow of the Frankfurt Association for the Advancement of Fundamental Research in Physics, Faculty of Physics, University of Frankfurt, Germany.

Subsequently Hardy shows that $\gamma$, i.e. the probability of entanglement is maximum when [2]

$$
|\beta|=0.9070
$$

and

$$
|\beta|=0.4211 .
$$

Being substantially larger than $\gamma=0$, this is a clear proof for the refutation of naïve classical realism. Apart of being rigorous, this result was experimentally verified many times. Despite its almost perfection and lucidity something went unnoticed in Hardy's paper due to his rounding of the numerics involved, for instance looking at the untruncated exact expressions [7] for $\alpha$ and $\beta$ namely

$$
|\alpha|=09069996487
$$

and

$$
|\beta|=0.4211313776
$$


we notice these are all golden mean functions with definite connections to the fundamental equations of E-infinity Cantorian spacetime theory. For instance [3-7]

$$
\begin{aligned}
& |\alpha||\beta|=\phi^{2}, \\
& |\alpha|^{2}+|\beta|^{2}-|\alpha||\beta|=\phi, \\
& (5 \sqrt{5}-11) 2=\phi^{5},
\end{aligned}
$$

and $[3,4]$

$$
(\alpha-\beta)^{2}=\phi^{3},
$$

where $\phi=(\sqrt{5}-1) / 2$ is the golden mean [3-7].

It is now an almost trivial matter for those familiar with A. Connes noncommutative Penrose fractal tiling [3] or E-infinity theory [3-7] to surmise that Hardy's quantum entanglement probability is a consequence of the Cantorian-fractal structure of micro spacetime topology [3-7]. In particular the fact that the Hausdorff dimension of the zero set is the golden mean $\phi$ and that of the empty set is $\phi^{2}$ is the reason for the appearance of the golden mean in Hardy's Gedanken experiment [2]. It is the purpose of the present paper to make this fundamental connection with far reaching consequences for quantum physics as crystal clear as possible.

\section{Derivation of Hardy's Quantum Entanglement Using Pure Logic and a Transfinite Probability Theory}

In some of his deepest papers Hardy recently called for the essential need for a probability theory which is both discrete and continuous at the same time but of course in different senses. This is essentially and indirectly echoing the same sentiment expressed by the present author long ago using the language of transfinite set theory [3-4]. Indeed in E-infinity theory we use Cantor sets which are totally disjointed and discrete and yet they have the cardinality of the continuum $[3,4]$. Combinatoric probability can only be finite and rational. Irrational probability exists only for geometrical probability which we cannot use or for topological (Hausdorff) probability which we do use [3-4]. This complies with what Hardy called for [3-7].

Let us start our logical analysis by systematically denying the existence of any meaning for spatial separation. We consider two particles or two different points in some space to be defined later on by the output of our requirements and analysis. The probability to be at point 1 will be denoted $d_{1}$ while the probability of being at point 2 is $d_{2}$. Consequently the probability of not being at 1 is obviously $1-d_{1}$ and similarly not being at 2 is $1-d_{2}$.
Let us create the maximum local muddle possible resulting from denying classical common sense and calculate the total probability of being all of the above at the same time. In other words, the probability of being in 1 and not in 1 as well as in 2 and not in 2 in addition to being in all of that at the same time. Following the multiplication theorem or the intersection rule, the total entanglement probability would be

$$
\mathrm{P}_{1}=d_{1}\left(1-d_{1}\right) d_{2}\left(1-d_{2}\right)
$$

To appreciate the value of $\mathrm{P}_{1}$ it has to be compared with the simplest local realism. To obtain in an analogous way the probability for simple local realism, we reason that this must negate being at 1 and at 2 at the same time. This non-entangled state is clearly $1-d_{1} d_{2}$ and must be substantially larger than $\mathrm{P}_{1}$. Thus we have established $\mathrm{P}_{2}$ of non-entanglement, namely

$$
\mathrm{P}_{2}=1-d_{1} d_{2}
$$

and consequently we can work with a "relative" probability defined for two particles in the most general way possible, namely

$$
\mathrm{P}=\mathrm{P}_{1} / \mathrm{P}_{2}
$$

or

$$
\mathrm{P}=\frac{d_{1}\left(1-d_{1}\right) d_{2}\left(1-d_{2}\right)}{\left(1-d_{1} d_{2}\right)}
$$

The next step is crucial because we are searching for an extremum for $\mathrm{P}$ which will turn out to be a maximum. This maximum is defined by

$$
\frac{\partial \mathrm{P}}{\partial d_{1}}=0
$$

and

$$
\frac{\partial \mathrm{P}}{\partial d_{2}}=0 \text {. }
$$

It is easily shown that the two equations result in one cubic algabraic equation with three solutions, namely for $d_{1}=d_{2}=1,-1 / \phi$ and $\phi$.

The third solution $d=\phi=0.61833989$ is clearly a confirmation of the E-infinity theory result where $d_{c}^{(0)}$ $=\phi$ is both the Hausdorff dimension of a random triadic Cantor set as well as being the topological probability of finding a Cantorian point in this set $[3,4]$. To obtain Hardy's result explicitly we insert $d_{1}=d_{2}=\phi$ in P and find [7]

$$
\mathrm{P}=\phi^{5}
$$

exactly as found by Hardy [2]. In a sense E-infinity is the limit set of the quantum geometry corresponding to quantum mechanics [3-7]. 


\section{Derivation of Hardy's Result Using E-Infinity Theory.}

Those familiar with E-infinity theory for which two convenient summaries and reviews may be found in $[3,4]$ know that the probability of finding a point in this space is $\phi^{3}$. This is the inverse of its average Hausdorff dimension $4+\phi^{3}$. The general formula for the dimension is $[3,4]$

$$
\langle n\rangle=\frac{1+d_{c}^{(0)}}{1-d_{c}^{(0)}}
$$

Consequently the probability is [3,7]

$$
1 /\langle n\rangle=\frac{1-d_{c}^{(0)}}{1+d_{c}^{(0)}} .
$$

This probability may be regarded as the result of "counterfactual" influence [1,2,7]. To find the total probability of two entangled points in this space we recall first that for each point on its own, the probability not counting "counterfactual" is $d_{c}^{(0)}[3,4]$. Consequently the entanglement not counting counterfactual is $\left(d_{c}^{(0)}\right)^{2}$. The total probability is consequently the product of $1 /\langle n\rangle$ with $\left(d_{c}^{(0)}\right)^{2}$ which means [7]

$$
\mathrm{P}=\left(d_{c}^{(0)}\right)^{2}\left(\frac{1-d_{c}^{(0)}}{1+d_{c}^{(0)}}\right) .
$$

Now either we trust the E-infinity result that $d_{c}^{(0)}=\phi$ or we maximize $\mathrm{P}$ with respect to $d_{c}^{(0)}$ and find a quadratic equation

$$
\left(d_{c}^{(0)}\right)^{2}+d_{c}^{(0)}-1=0
$$

from which we obtain two solutions

$$
d_{c}^{(0)}=\phi \text { and } d_{c}^{(0)}=\frac{1}{\phi}
$$
$[2,7]$

Inserting $d_{c}^{(0)}=\phi$ in $\mathrm{P}$ we find Hardy's result again

$$
\mathrm{P}=\phi^{5}
$$

To obtain the result $\mathrm{P}=0$ befitting the classical expectation of classical mechanics we just need to set $d_{c}^{(0)}=d_{c}^{(1)}=1$ of a classical one dimensional continuous line rather than a Cantor transfinite set of points in our symmetric expression for $\mathrm{P}$.

From the preceding derivation and unlike the first derivation, it is absolutely clear that Hardy's result is geometrically and topologically rooted in the Cantorian nature of micro quantum spacetime [3-7]. Consequently quantum entanglement is not counter intuitive but rather intuitive when we adopt the appropriate intuition of the zero measure transfinite point set geometry of Cantorian geometry [3-7].

\section{Connection between the Logical and the Topological Derivations of Hardy's Quantum Entanglement}

Let us return to the general $\mathrm{P}$ obtained from nonlocality logic

$$
\mathrm{P}=\frac{\left(d_{1}\right)\left(1-d_{1}\right)\left(d_{2}\right)\left(1-d_{2}\right)}{1-d_{1} d_{2}}
$$

Let us make the solution totally symmetric by setting

$$
d_{1}=d_{2}=d
$$

then we find

$$
\mathrm{P}=\frac{(d)^{2}(1-d)^{2}}{1-d^{2}}
$$

This means

$$
\mathrm{P}=(d)^{2} \frac{(1-d)^{2}}{(1-d)(1+d)}=(d)^{2} \frac{(1-d)}{(1+d)} .
$$

Not surprisingly, setting $d=d_{c}^{(0)}$ we find our formula for P obtained using E-infinity namely [7]

$$
\mathrm{P}=\left(d_{c}^{(0)}\right)^{2} \frac{1-d_{c}^{(0)}}{1+d_{c}^{(0)}}
$$

In fact we can generalize $\mathrm{P}$ for $\mathrm{n}$ particles or locations easily by writing [7]

$$
\mathrm{P}=\left(d_{c}^{(0)}\right)^{n} \frac{1-d_{c}^{(0)}}{1+d_{c}^{(0)}}
$$

where $n=2,3, \cdots$.

\section{Discussion and Conclusion}

A random Cantor set with its golden mean Hausdorff dimension interpreted as a topological Hausdorff probability is a synthetic a priori for a topology and a corresponding probability theory which unites the ununiteable, namely the discrete and the continuum $[3,4]$.

A Cantor set has a zero Lebesgue measure. That means zero length. In a sense it is physically not there and yet it has a substantial Hausdorff dimension, namely $\phi=0.618033$ [3-7]. Therefore it is 'there' to consider and work with. A Cantor set, in a way surprising to the naïve intuition, is there and not there at the same time. Measure zero and the emptiness of an empty set are aspects which quantum mechanics does not address directly. E-infinity is based on these subtle concepts [3-7]. Hardy's magnificent work [2] reached in a formal way using orthodox quantum mechanics the same result 
which we demonstrated to be natural and non spooky once suitable mathematics and the associated Cantorian based geometry are utilized to interpret and understand the results.

\section{References}

[1] J. S. Bell, "Speakable and Unspeakable in Quantum Mechanics,” Cambridge, 1987.

[2] L. Hardy, "Nonlocality for Two Particles without Inequalities,” Physical Review Letters, Vol. 71 No. 11, 1993, pp. 1665-1668. doi:10.1103/PhysRevLett.71.1665

[3] M. S. El Naschie, "A Review of E-Infinity Theory and the Mass Spectrum of High Energy Particle Physics," Chaos, Solitons \& Fractals, Vol. 19, No. 1, 2004, pp. 209-236.
[4] M. S. El Naschie, "The Theory of Cantorian Spacetime and High Energy Particle Physics (an Informal Review)," Chaos, Solitons \& Fractals, Vol. 41, No. 5, 2009, pp. 2635-2646. doi:10.1016/j.chaos.2008.09.059

[5] J.-H. He, et al., "The Importance of the Empty Set and Noncommutative Geometry in Underpinning the Foundations of Quantum Physics,” Nonlinear Science Letters B, Vol. 1, No. 1, 2001, pp. 15-24.

[6] M. S. El Naschie, "Quantum Collapse of Wave Interference Pattern in the Two-Slit Experiment: A Set Theoretical Resolution,” Nonlinear Science Letters A, Vol. 2, No. 1, 2011, pp. 1-9.

[7] J.-H. He, et al., "Quantum Golden Mean Entanglement Test as the Signature of the Fractality of Micro Spacetime,” Nonlinear Science Letters B, Vol. 1, No. 2, 2011, pp. 45-50. 